\title{
Comment on EAACI guideline: Anaphylaxis (2021 update)
}

\author{
Stefano Miceli Sopo ${ }^{1}$, Dario Sinatti ${ }^{1}$, Francesco Mastellone ${ }^{1}$, Giulia Bersani ${ }^{1}$, and \\ Mariannita Gelsomino ${ }^{1}$ \\ ${ }^{1}$ Fondazione Policlinico Universitario Agostino Gemelli IRCCS
}

January 28, 2022

\section{Comment on EAACI guideline: Anaphylaxis (2021 update)}

\section{Authors :}

Stefano Miceli Sopo ${ }^{\mathrm{a}}$, Dario Sinatti ${ }^{\mathrm{a}}$, Francesco Mastellone ${ }^{\mathrm{b}}$, Giulia Bersani ${ }^{\mathrm{a}}$, Mariannita Gelsomino ${ }^{\mathrm{b}}$.

\section{Affiliations:}

${ }^{a}$ Pediatric Allergy Unit, Pediatrics Section, Department of Life Sciences and Public Health, Policlinico Gemelli Universitary Foundation IRCCS, Catholic University of Sacre Hearth, Rome 00168, Italy.

b Post-Graduate School of Pediatrics, Department of Life Sciences and Public Health, Policlinico Gemelli Universitary Foundation IRCCS, Catholic University of Sacre Hearth, Rome 00168, Italy.

\section{Correspondence :}

\section{Stefano Miceli Sopo}

Pediatric Allergy Unit, Pediatrics Section, Department of Life Sciences and Public Health, Policlinico Gemelli University Foundation IRCCS, Catholic University of Sacred Heart, Rome, Italy.

Email: stefano.micelisopo@unicatt.it

Acknowledgements : not applicable.

Abstract : not applicable.

Keywords : allergy treatment, anaphylaxis, pediatrics

\section{Author contributions}

Stefano Miceli Sopo conceived design of the study and drafted the first version of the article. Material preparation and data collection were performed by Dario Sinatti, Francesco Mastellone, Giulia Bersani and Mariannita Gelsomino. All authors revised critically, drafted and approved the final manuscript as submitted and agreed to be accountable for all aspects of the work.

To the Editor

We have read with great interest the article by Muraro et $\mathrm{al}^{1}$ which deals with the update of the EAACI guideline on anaphylaxis. In their box 4 , the authors ${ }^{1}$ divide the indications for the prescription of selfinjectable adrenaline into two categories: a) "Absolute indications for adrenaline autoinjectors"; b) "Consider prescribing adrenaline autoinjectors". In this second category, the authors included oral immunotherapy (OIT) for food allergy (FA), explaining that anaphylaxis is a known adverse effect of OIT for food allergy and supporting this categorization by citing the EAACI guidelines on allergen immunotherapy: IgE-mediated food allergy of $2018^{2}$. However, in this last guideline ${ }^{2}$ and in particular in the box 7 ("General considerations 
before initiating FA-AIT") we read "Patients and their families should be motivated, adherent, and capable of administering emergency treatment (including intramuscular adrenaline) in case of adverse effects". In addition, in the box $12^{2}$ ("Summary of the management") we read that, in addition to other measures, the physician who decides to start an OIT for AF must not forget the "Provision of emergency kit with copy of emergency action plan and adrenaline auto-injector for treatment of anaphylaxis".

It would seem, in conclusion, that the EAACI 2018 guideline considers the prescription of the adrenaline auto-injector in case of OIT for FA to be essential, while the EAACI 2021 guideline considers it optional, to be taken into consideration, to think about it, but certainly not to be placed among the absolute indications. The change of this recommendation appears inexplicable, especially by the same scientific society, with some authors signing both documents and with the choice to support this variation with a reference that expresses itself differently.

Moreover, if we consider recent evidences in this regard, they seem to adopt the maximum prudence. The systematic review of Chu et $\mathrm{al}^{3}$ concludes: "In patients with peanut allergy, high-certainty evidence shows that available peanut oral immunotherapy regimens considerably increase allergic and anaphylactic reactions over avoidance or placebo".

\section{Conclusion}

In box 4 of the EAACI guideline on anaphylaxis ${ }^{1}$, we would consider moving the OIT for FA from the category "Consider prescribing adrenaline autoinjectors" to the category "Absolute indications for adrenaline autoinjectors". Moreover, we would put it as the first indication.

\section{References}

1. Muraro A, Worm M, Alviani C, et al. EAACI guideline: Anaphylaxis (2021 update). Allergy. 2021 Aug 3.

2. Pajno GB, Fernandez-Rivas M, Arasi S, et al. EAACI Guidelines on allergen immunotherapy: IgEmediated food allergy. Allergy. 2018;73(4):799-815.

3. Chu DK, Wood RA, French S, et al. Oral immunotherapy for peanut allergy (PACE): a systematic review and meta-analysis of efficacy and safety. Lancet. 2019 Jun 1;393(10187):2222-2232.

Stefano Miceli Sopo ${ }^{\text {a }}$ Prof

Dario Sinatti a MD

Francesco Mastellone ${ }^{\mathrm{b}}$ MD

Giulia Bersani a MD

Mariannita Gelsomino ${ }^{\mathrm{b}} \mathrm{MD}$.

${ }^{a}$ Pediatric Allergy Unit, Pediatrics Section, Department of Life Sciences and Public Health, Policlinico Gemelli Universitary Foundation IRCCS, Catholic University of Sacre Hearth, Rome 00168, Italy.

b Post-Graduate School of Pediatrics, Department of Life Sciences and Public Health, Policlinico Gemelli Universitary Foundation IRCCS, Catholic University of Sacre Hearth, Rome 00168, Italy.

Conflict of interests : the authors declare that they have no conflict of interests to disclose in relation to this paper.

Financial support : this research did not receive any specific grant from funding agencies in the public, commercial, or non-profit sectors. 